\title{
Medium Access Control with Channel State Information for Large Sensor Networks
}

\author{
Srihari Adireddy and Lang Tong \\ School of Electrical and Computer Engineering \\ Cornell University \\ Ithaca, NY 14853 \\ \{srihari,ltong\}@ece.cornell.edu
}

\begin{abstract}
Traditionally, random access protocols have been designed and studied by assuming simple models for the physical layer. We introduce a reception model that incorporates the channel states of the transmitting users and allows for multiple simultaneous successes. We assume that each user has access to his channel state and propose a variant of the Slotted ALOHA protocol for medium access where the transmit probability is chosen as a function of the channel state. We introduce the notion of asymptotic stable throughput and characterize the achievable asymptotic stable throughput through the use of channel state information. As an example, we consider the application of the results to sensor networks.
\end{abstract}

Index Terms-Multi-user Diversity, Channel State Information, Transmission Control, Large Scale Sensor Networks, Reachback Problem.

\section{INTRODUCTION}

The growth in the demand for data rate over wireless channels and the emergence of new networks like the sensor networks has led to a rethinking of the traditional network architecture and design principles. Cross layer design, where information is exchanged between layers to enhance performance, is being explored as an alternative to the traditional design paradigm [17]. In this context, allowing interaction between MAC and PHY layers seems natural, especially for wireless networks.

As has been noted in [5], the field of random access uses rather simplistic models for the physical layer. As the sophistication of signal processing increases, it is necessary to consider more accurate models of the physical layer and redesign the MAC layer based on these models so as to improve the overall performance of the network. One such model is the MPR model that was introduced in [6]. A shortcoming of this model is that it assumes that all users are identical and they behave in an identical manner in each slot. This assumption is not valid for wireless networks where different users experience different fading conditions. In fact, in a recent line of work, it has been shown that the knowledge of the fading channel at the transmitter can crucially change the resource allocation problem for multi-access fading channels [11], [4], [8].

There has been very little prior work in incorporating the channel knowledge into the random access protocols. We have

This work was supported in part by the Multidisciplinary University Research Initiative (MURI) under the Office of Naval Research Contract N00014-00-1-0564 and the ARL CTA on Communications and Networks under Grant DAAD19-01-2-011. previously introduced a reception model that takes into account the channel states of the transmitting users and also allows for a detailed abstraction of the physical layer processing [13]. In [13], we assumed decentralized channel state information (CSI), that is, each user has access to his channel state alone. We proposed that a variation of Slotted ALOHA be employed, where the transmit probability of each user is a function of the channel state in that particular slot. This function is called scheduler. We also assumed that the channel state varies independently and identically from slot to slot and from user to user. We then derived the maximum stable throughput (see [18]) of a finite user symmetric system as a function of the reception model and the scheduler employed. Optimal schedulers were obtained for some simple reception models [13]. It turns out that obtaining optimal scheduler for complicated reception models is in general a hard problem.

In this paper, we introduce the notion of asymptotic stable throughput (AST), which is the maximum stable throughput as the number of users goes to infinity while keeping the total input rate constant. Such a metric is of clear importance for large sensor networks and it also turns out to be simpler to obtain schedulers that are "good" based on AST. An important insight of this work is that the scheduler can be used to change the conditional distribution of the channel state as observed at the receiver. It is therefore beneficial to choose those schedulers that lead to conditional distributions that are good for the reception model under consideration.

The use of schedulers which use channel state is studied for the reachback problem in sensor networks. We assume that the network employs CDMA for the physical layer and the receiver uses a linear MMSE multi-user receiver to demodulate the users. The context of CDMA networks provides us with a particular reception model for which the theory can be applied. This application demonstrates the power of using CSI to vary the transmit probability.

Design of retransmission probability was considered in [10], [3], [19] but the concern was to make the protocol fair to the users. In [16], the retransmission probabilities are designed in order to maximize the throughput but the reception model was a simple one and centralized CSI was assumed. Slotted ALOHA where mobiles have the knowledge of the uplink SNR was considered in [20], [1]. In [20], the channel assumed was a collision channel and the knowledge of channel state was used to consider a particular power transmission policy. It was shown 
that because of power variation, the throughput increases with the number of users. In [1], a particular simple transmission scheme was chosen where the mobiles are allowed to transmit only above a certain threshold. The performance was evaluated by simulation and the threshold was not optimized but set in a heuristic fashion. Stability analysis for capture model was considered in [9]. It was not assumed that the nodes have access to their channel state information. The retransmission probabilities of different users was therefore kept fixed.

In Section II, we describe the system model in detail. In Section III, we introduce the describe the notion of maximum stable throughput (MST) and give expressions for MST as a function of the control and the reception model. In Section IV, we introduce the notion of asymptotic stable throughput and gives expressions for achievable AST. In Section V we apply the theory to the problem of reachback in sensor networks and we list our concluding remarks in Section VI.

\section{SySTEM MODEL}

We consider a network where $M$ users are trying to communicate with a base station over a common channel. Each user has a buffer of infinite length that stores the incoming packets until they are sent successfully to the base station. Time is slotted into intervals of equal length that is equal to the time required to transmit a packet. We make the slot time equal to one time unit and slot $t$ is assumed to occupy the time $[t, t+1)$. We denote by $X_{m}^{(t)}$ the number of incoming packets to user $m$ during time slot $t$. The packet arrival process for different $X_{m}^{(t)}$ for $m=1, \cdots, M$ and $t=0,1, \cdots$ is assumed to be independent and identically distributed as well. The arrival process has a finite mean $\frac{\lambda}{M}$ and finite variance. The above model for the arrival process is the same as that in [18] for a symmetric system.

The channel between the $m^{\text {th }}$ user and the base station during slot $t$ is parametrized by $\gamma_{m}^{(t)}$. It is assumed that the quantities $\gamma_{m}^{(t)}$ for $m=1, \cdots, M$ and $t=0,1, \cdots$ are independent and identically distributed with probability distribution $F(\gamma)$. Further, we assume that the user $m$ has access to the uplink CSI $\gamma_{m}^{(t)}$ at time $t$.

We define a general reception model that is given by a set of $M$ functions where given $\Theta_{k}=\left(\theta_{k}^{(1)}, \cdots, \theta_{k}^{(k)}\right)$, a binary $k$-tuple, the $k^{\text {th }}$ function $\Phi^{(k)}\left(\gamma_{1}, \cdots, \gamma_{k} ; \Theta_{k}\right)$ gives the probability of outcome $\Theta_{k}$ (users corresponding to indices with one are successful) when $k$ users whose CSI is given by $\gamma=$ $\left(\gamma_{1}, \cdots, \gamma_{k}\right)$ transmit. That is,

$\Phi^{(k)}\left(\gamma_{1}, \cdots, \gamma_{k} ; \Theta_{k}\right)=\operatorname{Pr}\left\{\Theta_{k} \mid k\right.$ users tx, $\left.\gamma=\left(\gamma_{1}, \cdots, \gamma_{k}\right)\right\}$.

We define $\Psi^{(k)}\left(\gamma_{1}, \cdots, \gamma_{k}\right)$ as

$\Psi^{(k)}\left(\gamma_{1}, \cdots, \gamma_{k}\right)=\sum_{i=1}^{k} \mathrm{E}\left\{\theta_{k}^{(i)} \mid k\right.$ users tx, $\left.\gamma=\left(\gamma_{1}, \cdots, \gamma_{k}\right)\right\}$

the expected number of packets successfully demodulated when the CSI of the transmitting users is $\left(\gamma_{1}, \cdots, \gamma_{k}\right)$. Given a distribution function $F(\cdot)$, we define $C_{k}(F(\cdot))$ as the expected number of packets received conditioned on $k$ users transmit and their CSI is distributed i.i.d according to $F(\cdot)$. That is

$$
C_{k}(F(\cdot))=\sum_{i=1}^{k} \mathrm{E}\left\{\theta_{k}^{(i)} \mid k \text { users tx }\right\}
$$

Note that this reception model allows the reception of multiple packets simultaneously.

We assume that for any given $\left(\gamma_{1}, \cdots, \gamma_{k}\right)$, adding an extra user decreases the probability of packets success for each of the $k$ users. We also assume that for each $k$, if we permute the SNRs $\left(\gamma_{1}, \cdots, \gamma_{k}\right)$ and apply the same permutation to the bits of $\Theta_{k}$, the value of $\Phi^{(k)}(\cdots)$ does not change. Note that $\gamma^{(m)}(t)$ can be used to model various parameters that influence the reception. Examples include physical channel gain, position of the mobile etc.

In a conventional ALOHA system [18], if the user $m$ has a packet to transmit, he transmits it with a probability $p_{m}$. We consider a more general random access scheme, where the probability of transmission for each user is allowed to be a function of his CSI. The function is called the scheduler and is denoted by $s(\cdot)$. Thus we assume that in slot $t$, user $m$ transmits a packet with a probability $s\left(\gamma_{m}^{(t)}\right)$. At the end of slot $t$, the base station broadcasts the indexes of those users whose packets it was able to demodulate successfully.

\section{Maximum Stable Throughrut}

In this section, we give expressions for the maximum stable throughput of the system described in the previous section. Let the $M$-tuple $\mathbf{N}^{(t)}=\left(N_{1}^{(t)}, N_{2}^{(t)}, \cdots, N_{M}^{(t)}\right)$ be the length of the buffers at each node at the beginning of slot $t$. We say that the system is stable for a particular arrival process, if for $\mathbf{x} \in \mathbb{N}_{+}^{M}$

$$
\lim _{t \rightarrow \infty} \operatorname{Pr}\left\{\mathbf{N}^{(t)}<\mathbf{x}\right\}=H(\mathbf{x}) \quad \lim _{\mathbf{x} \rightarrow \infty} H(\mathbf{x})=1
$$

where $\mathbb{N}_{+}$is the set of non-negative integers. This notion of stability is also used in [18]. The maximum stable throughput is defined as the supremum of all input rates $\lambda$ for which the system is stable. The arguments in [18] were extended in [13] to derive the following theorem.

Theorem 1: Given the density function of uplink SNR $F(\gamma)$, the scheduler $s(\gamma)$ and the reception functions $\left\{\Phi^{(k)}(\cdots)\right\}_{k=1}^{M}$, the maximum stable throughput is given by

$$
\lambda^{*}(s(\cdot))=\sum_{k=1}^{n}\left(\begin{array}{l}
n \\
k
\end{array}\right)\left(1-p_{s}\right)^{n-k} p_{s}^{k} C_{k}\left(G_{s}(\cdot)\right) .
$$

where $p_{s}=\int s d F$ and the distribution $G_{s}(\cdot)$ is defined as

Proof: Refer to [13].

$$
G_{s}(\gamma)=\frac{\int_{0}^{\gamma} s(x) d F(x)}{p_{s}}
$$

It is important to derive optimal schedulers for various reception models by maximizing the MST of the system. Unfortunately, it is not possible to obtain such schedulers except for some simple reception models. Some examples can be found in [13]. The difficulty comes from the fact that the schedulers affect both the probability of transmission $p_{s}$ and the a posteriori CSI distribution $G_{s}(\cdot)$. 


\section{Asymptotic Stable Throughrut}

In this section, we introduce the notion of asymptotic stable throughput (AST). Essentially, this is the maximum stable throughput for "large" networks. It turns out that it is easier to find schedulers that are optimal with respect to AST.

Definition 1: Given the distribution function of CSI $F(\gamma)$, the scheduler sequence $s_{n}(\gamma)$ and the reception functions $\left\{\Phi^{(k)}(\cdots)\right\}_{k=1}^{M}$, the asymptotic stable throughput is defined as $\lambda^{*}\left(\left\{s_{n}(\cdot)\right\}\right) \triangleq \liminf _{n \rightarrow \infty} \sum_{k=1}^{n}\left(\begin{array}{l}n \\ k\end{array}\right)\left(1-p_{s_{n}}\right)^{n-k} p_{s_{n}}^{k} C_{k}\left(G_{s_{n}}(\cdot)\right)$.

where $p_{s_{n}}=\int s_{n} d F$ and the distribution $G_{s_{n}}(\cdot)$ is defined as

$$
G_{s_{n}}(\gamma)=\frac{\int_{0}^{\gamma} s_{n}(x) d F(x)}{p_{s_{n}}}
$$

It should be noted that the distribution function $G_{s_{n}}(\gamma)$ is the distribution of CSI conditioned on the event that a user transmitted. It should be apparent that the reception is affected by the a posteriori CSI distribution rather than $F(\gamma)$.

We first have the AST for the case when the nodes do not use CSI.

Proposition 1: If the sequence of scheduler $s_{n}(\gamma)$ is chosen to be independent of $\gamma$ but a function of $n$ alone, then the maximum possible AST is given by

$$
\lambda_{t}^{*}=\sup _{x} e^{-x} \sum_{k=1}^{\infty} \frac{x^{k}}{k !} C_{k}(F(\cdot)),
$$

where $F(\gamma)$ is the distribution of $\gamma$.

Proof: The proof follows from [7].

Thus, given $F(\gamma)$ and the reception model, $\lambda_{t}^{*}$ is the maximum achievable AST without using CSI.

It is possible to construct a simple sequence of schedulers that improves significantly upon the AST obtained above. Let $T(\cdot)$ be a distribution function such that $\mu_{F}(A)=0 \Rightarrow$ $\mu_{T}(A)=0$ (notation $T(\cdot) \ll F(\cdot)$ ), then from RadonNikodym theorem, there exists a non-negative function $\frac{d T}{d F}$ such that

$$
\mu_{T}(A)=\int_{A} \frac{d T}{d F} d F
$$

Proposition 2: With the sequence of schedulers chosen as

$$
s_{n}(\gamma)=\min \left(\frac{x}{n} \frac{d T}{d F}, 1\right)
$$

the asymptotic stable throughput is given by

$$
\lambda=e^{-x} \sum_{k=1}^{\infty} \frac{x^{k}}{k !} C_{k}(T(\cdot)) .
$$

Proof: Details in [12].

By comparing Proposition 1 with Proposition 2, it can be seen that the effect of the chosen sequence is to effectively change the CSI distribution from $F(\gamma)$ to $T(\gamma)$. Through a proper choice of scheduler sequence, it is therefore possible to achieve an AST of

$$
\lambda_{c}^{*}=\sup _{x, T(\cdot) \ll F(\cdot)} e^{-x} \sum_{k=1}^{\infty} \frac{x^{k}}{k !} C_{k}(T(\cdot)) .
$$

The quantity $\lambda_{c}^{*}$ is in some sense the capacity associated with reception model and $F(\cdot)$. For different reception models, it is important to characterize $\lambda_{c}^{*}$ and find distributions $T(\cdot)$ that achieve an AST that is close to $\lambda_{c}^{*}$.

\section{Reachback in Sensor Networks}

We will now apply the theory to the reachback problem in sensor networks. For us, reachback refers to the data gathering phase of the operation of sensor networks. This could, for example, be the scenario shown in Fig 1, where the airplane acts as the collecting station. The number of nodes in a sensor

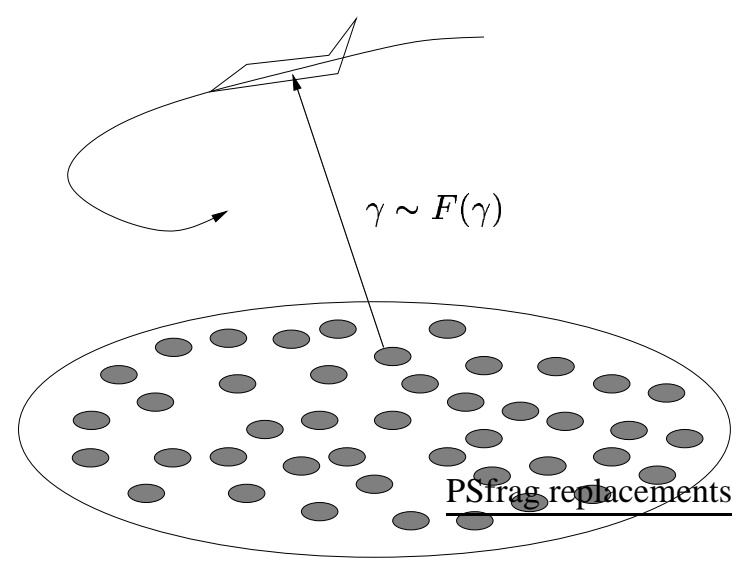

Fig. 1: Reachback in Sensor Networks

figure

network can be very large, some times in thousands. Thus the use of asymptotic stable throughput as a metric is justified here. But we should emphasize that the ideas presented are general and can be used in many settings.

We assume that the uplink of the sensor network employs CDMA and the collecting station uses a linear MMSE multiuser receiver to demodulate the users. (For the details about the reception model when a linear MMSE multi-user receiver is employed, please see [15].) The packet is successfully demodulated if after the front-end processing the signal to interference ratio is greater than $\beta$. The parameter $\beta$ is a function of modulation scheme used, code used and quality of service required for the application in terms of the bit error rate. We will study the case when the channel state is chosen to be the power that would be received from the mobile, if it transmits in that slot. This is the product of transmitted power $P_{T}$ and the propagation channel gain. Since we require that each user have access to his channel state, we imagine a time division duplex (TDD) system where the collecting station is transmitting a pilot tone. The received power from a node can be modeled as

$$
P_{R}=K R^{2} P_{T},
$$

where $K$ is a constant, $R$ is the propagation channel gain that is Rayleigh distributed, because all the nodes are typically at the same distance from the collecting station and undergo the same propagation loss and shadow fading. Thus the underlying CSI distribution can be assumed to be exponential.

Fig 2 shows the variation of AST with respect to $x$, a design parameter that is equal to the average number of packets 
transmitted in each slot. The solid line corresponds to the case when CSI is not used for transmission control and therefore the a posteriori CSI is exponential. If the transmission control employs CSI, it is possible to manipulate the CSI distribution. "Good" target distributions for this model are those with a rolloff between 0 and 1. As in [2], given a distribution function $G(\cdot)$, define $\delta$ to be the roll-off $G(\cdot)$, if there exists a $c$ such that $0<c<\infty$ and

$$
\lim _{\gamma \rightarrow \infty}(1-G(\gamma)) \gamma^{\delta}=c
$$

(See [14] for an explanation of why we choose these distributions for target distributions.) The transmission control that changes the a posteriori distribution to one with a roll-off $\delta$ is

$$
s_{n}(\gamma)=\min \left(\frac{e^{\frac{\gamma}{P_{T}}}}{\gamma^{1+\delta}}, 1\right) 1_{\gamma>\gamma_{0}}
$$

where $\gamma_{0}$ is any constant. Figure 2 shows the gain obtained by

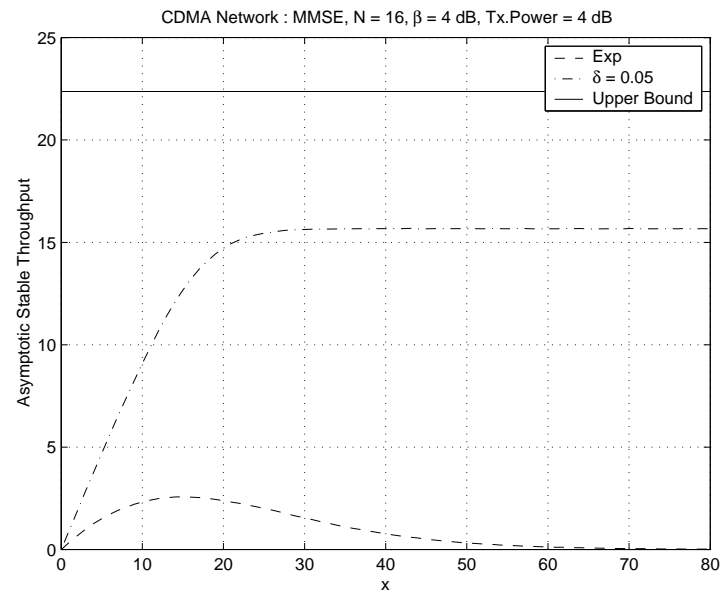

Fig. 2: Linear MMSE Multi-user Receiver

figure

going from exponential distribution to a distributions with $\delta=$ 0.05. For a fixed AST, the required $x$ is smaller when CSI is used which implies that the average number of transmissions is smaller for the protocol proposed and this fact has implications on the network wide power savings.

It is possible to achieve an AST of $N$ using distributions with a roll-off. ( assuming $\beta>1$, which is typical). However, distributions that can be used to improve the AST beyond $N$ and the capacity $\lambda_{c}^{*}$ are not known. The following theorem summarizes the importance of CSI for the reception model under consideration.

Theorem 2: Assume $\beta>1$ and $F(\gamma)=1-e^{-\frac{\gamma}{P_{T}}}$, then

$$
\lim _{P_{T} \rightarrow 0} \lambda_{t}^{*}=0 .
$$

However, for any given $P_{T}$, the maximum achievable AST with CSI satisfies

$$
N \leq \lambda_{c}^{*} \leq N+\frac{N}{\beta} .
$$

Thus, without CSI the achievable AST goes to zero with the transmit power $P_{T}$ but using CSI, it is theoretically possible to achieve an AST at least as big $N$, even with arbitrarily small power.

\section{CONCLUSIONS}

In this paper, we considered the use of channel state information to vary the transmit probability of Slotted ALOHA for large networks. We introduced the notion of asymptotic stable throughput (AST) and derived expressions for achievable AST. To illustrate the gains that are possible, we applied the proposed MAC protocol to a CDMA sensor network.

\section{REFERENCES}

[1] A.Jahn and A.Bottcher. Improvement of slotted Aloha for land-mobile satellite communications, using channel state information. In IEEE Vehicular Technology Conference, pages 339-42, May 1993.

[2] B.Hajek, A.Krishna, and R.O.LaMaire. "On the Capture Probability for a Large Number of Stations". IEEE Trans. Communications, 45(2):254260, February 1997.

[3] B.Wu and Q.Wang. "Maximization of the channel utilization in wireless heterogeneous multiaccess networks ". IEEE Transactions on Vehicular Technology, 46(2):437-44, May 1997.

[4] D.N.C.Tse and S.V.Hanly. Multiaccess Fading Channels : I Polymatroid Structure, Optimal Resource Allocation and Throughput Capacities . IEEE Trans. Information Theory, 44(7):2796-2815, November 1998.

[5] Anthony Ephremides and Bruce Hajek. Information Theory and Communication Networks: An Unconsummated Union. IEEE Trans. Inform. Theory, 44(6):2416-2434, October 1998.

[6] S. Ghez, S. Verdú, and S. Schwartz. Stability Properties of Slotted Aloha with Multipacket Reception Capability. IEEE Trans. Automatic Control, 33(7):640-649, July 1988.

[7] S. Ghez, S. Verdú, and S. C. Schwartz. "Optimal Decentralized Control in the Random Access Multipacket Channel”. IEEE Trans. Automat. Contr. 34(11):1153-1163, November 1989.

[8] I.E.Telatar and S.Shamai. Some information theoretic aspects of decentralized power control in multiple access fading channels. In Proc. Info. Theory and Networking Workshop, pages 23-23, Piscataway, NJ, 1999.

[9] J.Sant and V.Sharma. "Performance analysis of a slotted-ALOHA protocol on a capture channel with fading ". Queueing Systems, Theory and Applications, 34(1):1-35, 2000.

[10] T.Yamazato K.Mori, T.Kobayashi and A.Ogawa. "Adaptive transmit permission probability control in CDMA cellular packet communications with site diversity ". IEICE Transactions on Communications, E83B(7):1445-53, July 2000.

[11] R.Knopp and P.A.Humblet. Information capacity and power control in single cell multi-user communications . In Proc. Intl Conf. Comm., pages 331-335, Seattle, WA, June 1995.

[12] S.Adireddy and L.Tong. On the Use of Channel State Information for Random Access Networks : Part I Theory. In Preparation, August 2002.

[13] S.Adireddy and L.Tong. Optimal Transmission Probabilities for Slotted ALOHA in Fading Channels . In Proc. CISS'02, Princeton, NJ, March 2002.

[14] S.Adireddy and L.Tong. Transmission Control with Channel State Information for CDMA Networks. In Submitted to ICC'03, Anchorage, AL, August 2002.

[15] S.Adireddy and L.Tong. Utilizing Channel Knowledge for Transmission Control in Slotted ALOHA. In To be Submitted to ISIT'03, Yokohoma, Japan, June 2003.

[16] J.A.Silvester T.-K.Liu and A.Polydoros. "Retransmission control and fairness issue in mobile slotted ALOHA networks with fading and nearfar effect ". Mobile Networks and Applications, 2(1):101-10, 1997.

[17] L. Tong, Q. Zhao, and G. Mergen. Multipacket reception in random access wireless networks: from signal processing to optimal medium access control. IEEE Communication Magazine, 39(12):108-112, Nov 2001. Special issue on Design Methodologies for Adaptive and Multimedia Networks.

[18] B.S. Tsybakov and V.A. Mikhailov. Ergodicity of Slotted Aloha System. Problemy Peredachi Informatsii, 15(4):73-87, Oct-Dec 1979.

[19] J.Ward W.Ren and M.Sweeting. Capture effect and its enhancement in LEO satellite channel . In Proc. EUROCOMM'00, pages 184-188, May 2000 .

[20] X.Qin and R.Berry. "Exploiting Multiuser Diversity in Wireless ALOHA Networks". In Proc. Allerton Conf. on Communication, Control and Computing, Allerton, IL, October 2001. 